\title{
On the control of a nonlinear system of viscoelastic equations
}

\section{Sur le contrôle d'un système non-linéaire d'équations viscoélastiques}

\author{
Muhammad I. Mustafa \\ Department of Mathematics - University of Sharjah \\ P.O.Box 27272, Sharjah, United Arab Emirates \\ mmustafa@sharjah.ac.ae
}

ABSTRACT. In this paper we consider a nonlinear system of two coupled viscoelastic equations, prove the well posedness, and investigate the asymptotic behaviour of this system. We use minimal and general conditions on the relaxation functions and establish explicit energy decay formula which gives the best decay rates expected under this level of generality. Our new result generalizes the earlier related results in the literature.

AMS Classification: 35B40, 74D99, 93D15, 93D20.

KEYWORDS AND PHRASES. General Decay, Coupled System, Viscoelasticity, Relaxation function.

\section{Introduction}

In this paper, we are concerned with the following coupled system

$$
\begin{cases}u_{t t}-\Delta u+\int_{0}^{t} g_{1}(t-\tau) \Delta u(\tau) d \tau+f_{1}(u, v)=0, & \text { in } \Omega \times(0, \infty) \\ v_{t t}-\Delta v+\int_{0}^{t} g_{2}(t-\tau) \Delta v(\tau) d \tau+f_{2}(u, v)=0, & \text { in } \Omega \times(0, \infty) \\ u=v=0, \quad \text { on } \partial \Omega \times(0, \infty) & \\ u(\cdot, 0)=u_{0}, u_{t}(\cdot, 0)=u_{1}, v(\cdot, 0)=v_{0}, v_{t}(\cdot, 0)=v_{1}, & \text { in } \Omega,\end{cases}
$$

where $u$ and $v$ denote the transverse displacements of waves and $\Omega$ is an open bounded domain of $\mathbb{R}^{n}$ with a smooth boundary $\partial \Omega$. The relaxation functions $g_{1}, g_{2}$ and the nonlinearities $f_{1}, f_{2}$ will be specified later. System (1.1) arises in the theory of viscoelasticity and describes the interaction of two scalar fields. In [3], Andrade and Mognon considered a similar problem with the nonlinearities

$$
f_{1}(u, v)=|u|^{p-2} u|v|^{p} \quad \text { and } \quad f_{2}(u, v)=|v|^{p-2} v|u|^{p}
$$

where $p>1$ if $n=1,2$ and $1<p \leq \frac{n-1}{n-2}$ if $n \geq 3$. They proved the well posedness for the problem under restrictive assumptions on the relaxation functions. In [37], Santos considered the coupling

$$
f_{1}(u, v)=a(u-v) \quad \text { and } \quad f_{2}(u, v)=-a(u-v),
$$

where $a$ is a positive constant, and assumed that

$$
-a_{1} g_{i}^{p}(t) \leq g_{i}^{\prime}(t) \leq-a_{2} g_{i}^{p}(t) \quad \text { and } \quad 0 \leq g_{i}^{\prime \prime}(t) \leq \gamma g_{i}^{p}(t), \quad i=1,2,
$$

for some $1 \leq p<2$. He proved that when the kernels decay exponentially (resp. polynomially) the first- and the second-order energy of the solution decay exponentially (resp. polynomially). In [25], Messaoudi and Tatar used the following weaker conditions on the relaxation functions

$$
g_{i}^{\prime}(t) \leq-c_{i} g_{i}^{p_{i}}(t), \quad i=1,2,
$$


for some $1 \leq p_{1}, p_{2}<3 / 2$, and more general forms of nonlinearities. They proved an exponential decay for $\left(p_{1}, p_{2}\right)=(1,1)$ and a polynomial decay for $\left(p_{1}, p_{2}\right) \neq(1,1)$. Liu [17] used the same hypothesis for a quasilinear system and established uniform decay results.

The problem, with a single viscoelastic equation, has been extensively discussed by many authors. We refer to $[5,24,26,27,28]$ for subsequent results which proved that the energy decays exponentially (resp. polynomially) if the relaxation function $\mathrm{g}$ decays exponentially (resp.polynomially). The same results were obtained by Alabau-Boussouira et al. [2], Cannarsa et al. [6] and Rivera et al. [29,30] for more general abstract equations and by Cavalcanti and Oquendo [9] for equations with both viscoelastic and frictional damping terms..

Then, a natural question was raised: how does the energy behave as the kernel function does not necessarily decay polynomially or exponentially? Messaoudi [20,21] studied

$$
u_{t t}-\Delta u+\int_{0}^{t} g(t-\tau) \Delta u(\tau) d \tau=b|u|^{\gamma} u
$$

for $b=0$ and $b=1$ and considered relaxation functions satisfying

$$
g^{\prime}(t) \leq-\xi(t) g(t), t \geq 0
$$

where $\xi(t): \mathbb{R}_{+} \rightarrow \mathbb{R}_{+}$is a nonincreasing differentiable function with

$$
\left|\frac{\xi^{\prime}(t)}{\xi(t)}\right| \leq k
$$

for some constant $k$. He proved that the decay rate of the solution energy is similar to that of the relaxation function which is not necessarily of exponential or polynomial type. After that a series of papers using (1.2) and (1.3) has appeared, see for instance [11,16,23,32,35,38]. On the other hand, a condition of the form

$$
g^{\prime}(t) \leq-\chi(g(t))
$$

where $\chi$ is a convex function satisfying some smoothness properties, was introduced by AlabauBoussouira and Cannarsa [1] and used then by several authors with different approaches. We refer to $[7,10,13,34]$ where decay results in terms of $\chi$ were obtained, to Lasiecka and Wang [14] where not only general but also optimal result was established, and to Cavalcanti et al. [8] where new methodology and tricks were used to treat the interaction between the nonlinearity and viscoelasticity.

Motivated by these works, Liu [15] imposed the conditions (1.2) and (1.3) on $g_{1}, g_{2}$ in the coupled system (1.1) and improved the earlier result in [25]. Later on, Mustafa [33] treated (1.1) using only (1.2). The same was done by Hao and Cai [12], Messaoudi and Al-Gharabli [22], and Said-Houari et al. [36] for similar systems.

Our aim in this work is to investigate (1.1) for relaxation functions of more general type than the ones in (1.2) and (1.4), namely $g^{\prime}(t) \leq-\xi(t) \chi(g(t))$, where $\chi$ is increasing and convex without any additional constraints. Such a condition on $g$ was first used by Mustafa [31] for a single linear viscoelastic wave equation, and then used by Liu et al. [18,19] for a Moore-Gibson-Thompson equation with memory. 
Here, we use this condition, overcome the difficulty brought by the nonlinearities, and establish explicit energy decay formulas which give the best optimal decay rates expected under this level of generality and from which the usual exponential and polynomial decay rate estimates are only special cases. Our results improve and generalize the earlier related results in the literature. The proof is based on the multiplier method and makes use of some properties of convex functions. The paper is organized as follows. In section 2, we present our results. Then, in section 3, the well posedness of the problem is established. Some technical lemmas are provided in section 4. The proof of the main result is given in section 5 .

\section{The main results}

We consider the following hypotheses

(H1) $g_{i}:[0, \infty) \rightarrow(0, \infty)$ (for $\left.i=1,2\right)$ are differentiable functions satisfying

$$
1-\int_{0}^{\infty} g_{i}(s) d s=l_{i}>0,
$$

and there exists a $C^{1}$ function $H:(0, \infty) \rightarrow(0, \infty)$ which is linear or it is strictly increasing and strictly convex $C^{2}$ function on $(0, r], r<m_{0}=\min \left\{g_{1}(0), g_{2}(0)\right\}$, with $H(0)=H^{\prime}(0)=0$, such that

$$
g_{i}^{\prime}(t) \leq-\xi_{i}(t) H\left(g_{i}(t)\right), \quad(i=1,2) \quad \forall t \geq 0
$$

where $\xi_{1}, \xi_{2}$ are positive nonincreasing differentiable functions.

(H2) $f_{i}: \mathbb{R}^{2} \rightarrow \mathbb{R}$ (for $\left.i=1,2\right)$ are $C^{1}$ functions, with $f_{i}(0,0)=0$, and there exists a function $F$ such that

$$
\begin{aligned}
& f_{1}(x, y)=\frac{\partial F}{\partial x}, f_{2}(x, y)=\frac{\partial F}{\partial y} \\
& F \geq 0, \quad x f_{1}(x, y)+y f_{2}(x, y)-F(x, y) \geq 0,
\end{aligned}
$$

and

$$
\left|\frac{\partial f_{i}}{\partial x}(x, y)\right|+\left|\frac{\partial f_{i}}{\partial y}(x, y)\right| \leq d\left(1+|x|^{\beta_{i 1}-1}+|y|^{\beta_{i 2}-1}\right) \quad \forall(x, y) \in \mathbb{R}^{2}
$$

for some constant $d>0$ and $\beta_{i j} \geq 1,(n-2) \beta_{i j} \leq n$ for $i, j=1,2$.

We observe that assumption (H2) gives, for some positive constant $k$, that

$$
\left|f_{i}(x, y)\right| \leq k\left(|x|+|y|+|x|^{\beta_{i 1}}+|y|^{\beta_{i 2}}\right)
$$

and

$$
\left|f_{i}(x, y)-f_{i}(r, s)\right| \leq k\left(1+|x|^{\beta_{i 1}-1}+|y|^{\beta_{i 2}-1}+|r|^{\beta_{i 1}-1}+|s|^{\beta_{i 2}-1}\right)(|x-r|+|y-s|)
$$

for all $(x, y),(r, s) \in \mathbb{R}^{2}$ and $i=1,2$. Throughout this paper, $c$ is used to denote a generic positive constant. We will also be using the embedding $H_{0}^{1}(\Omega) \hookrightarrow L^{s}(\Omega)$ for $2 \leq s \leq 2 n /(n-2)$ if $n \geq 3$ or $s \geq 2$ if $n=1,2$; i.e., for any $\phi \in H_{0}^{1}(\Omega)$,

$$
\|\phi\|_{s} \leq c\|\nabla \phi\|_{2}
$$


Now, we introduce the energy functional

$$
\begin{aligned}
& E(t):=\frac{1}{2} \int_{\Omega}\left(u_{t}^{2}+\left(1-\int_{0}^{t} g_{1}(s) d s\right)|\nabla u|^{2}\right) d x+\frac{1}{2}\left(g_{1} \circ \nabla u\right)(t) \\
& +\frac{1}{2} \int_{\Omega}\left(v_{t}^{2}+\left(1-\int_{0}^{t} g_{2}(s) d s\right)|\nabla v|^{2}\right) d x+\frac{1}{2}\left(g_{2} \circ \nabla v\right)(t)+\int_{\Omega} F(u, v) d x,
\end{aligned}
$$

where

$$
\left(g_{i} \circ w\right)(t)=\int_{\Omega} \int_{0}^{t} g_{i}(t-\tau)|w(t)-w(\tau)|^{2} d \tau d x .
$$

For the well-posedness, we prove, in section 3 , the following result.

Theorem 2.1. Let $\left(u_{0}, u_{1}\right),\left(v_{0}, v_{1}\right) \in\left(H^{2}(\Omega) \cap H_{0}^{1}(\Omega)\right) \times H_{0}^{1}(\Omega)$ be given. Assume that (H1) and (H2) are satisfied, then problem (1.1) has a unique strong solution

$$
u, v \in L^{\infty}\left(\mathbb{R}_{+} ; H^{2}(\Omega) \cap H_{0}^{1}(\Omega)\right) \cap W^{1, \infty}\left(\mathbb{R}_{+} ; H_{0}^{1}(\Omega)\right) \cap W^{2, \infty}\left(\mathbb{R}_{+} ; L^{2}(\Omega)\right) .
$$

Our main stability result is the following.

Theorem 2.2. Assume that (H1) and (H2) hold. Then there exist positive constants $k_{1} \leq 1$ and $k_{2}$ such that, along the solution of (1.1), the energy functional satisfies

$$
E(t) \leq k_{2} H_{1}^{-1}\left(k_{1} \int_{t_{1}}^{t} \xi(s) d s\right)
$$

where

$$
H_{1}(t)=\int_{t}^{r} \frac{1}{s H^{\prime}(s)} d s \quad, \quad \xi(t)=\min \left\{\xi_{1}(t), \xi_{2}(t)\right\}
$$

and $t_{1}=\max \left\{g_{1}^{-1}(r), g_{2}^{-1}(r)\right\}$. Here, $H_{1}$ is strictly decreasing and convex on $(0, r]$, with $\lim _{t \rightarrow 0} H_{1}(t)=$ $+\infty$.

The proof of this Theorem will be given in section 5. As a diret application, the following corollary shows that the optimal exponential and polynomial decay rate estimates, already proved for $g_{i}$ satisfying (2.1) and $g_{i}^{\prime} \leq-a_{i} g_{i}^{p_{i}}, 1 \leq p_{1}, p_{2}<2$, are special cases of our result.

Corollary 2.3. Assume that $(H 1-H 2)$ hold where, for $i=1,2,(2.2)$ is given by

$$
g_{i}^{\prime}(t) \leq-\xi_{i}(t) g_{i}^{p_{i}}(t), \quad 1 \leq p_{i}<2
$$

Then there are positive constants $k, \bar{k}, k_{1}$ such that the decay rate of $E$ is given by

$$
E(t) \leq\left\{\begin{array}{cl}
k e^{-k_{1} \int_{0}^{t} \xi(s) d s}, & \text { if } p=1 \\
\bar{k}\left(1+\int_{0}^{t} \xi(s) d s\right)^{\frac{-1}{p-1}}, & \text { if } 1<p<2
\end{array} .\right.
$$

where $\xi(t)=\min \left\{\xi_{1}(t), \xi_{2}(t)\right\}$ and $p=\max \left\{p_{1}, p_{2}\right\}$. 
Proof. Here, with $M_{0}=\max \left\{g_{1}(0), g_{2}(0)\right\}$, we see that $g_{i}^{\prime}(t) \leq-M_{0}^{p} \xi_{i}(t) H\left(g_{i}(t)\right)$ where $H(s)=$ $\left(\frac{s}{M_{0}}\right)^{p}$ is linear for $p=1$, and strictly increasing and strictly convex on $\left(0, m_{0}\right]$ for $1<p<2$. Then (2.7) and simple calculations lead to (2.8).

\section{Remarks}

(1) It has to be noted that, in case $\int_{0}^{\infty} \xi(s) d s=\infty$, Theorem 2.2 ensures $\lim _{t \rightarrow \infty} E(t)=0$. Moreover, the decay rate of $E(t)$ driven by (2.7) is optimal in the sense that it is consistent with the decay rates of $g_{1}(t)$ and $g_{2}(t)$ driven by (2.2). In fact, making use of (2.2) for $i=1,2$ yields

$$
g_{i}^{\prime}(t) \leq-\xi_{i}(t) H\left(g_{i}(t)\right) \Longrightarrow \int_{g_{i}^{-1}(r)}^{t} \frac{-g_{i}^{\prime}(s)}{H\left(g_{i}(s)\right)} d s=\int_{g_{i}(t)}^{r} \frac{d s}{H(s)} \geq \int_{g_{i}^{-1}(r)}^{t} \xi_{i}(s) d s
$$

so, if we define $H_{0}(t)=\int_{t}^{r} \frac{1}{H(s)} d s$, then $H_{0}$ is strictly decreasing and convex on $(0, r]$, with $\lim _{t \rightarrow 0} H_{0}(t)=$ $+\infty$, and $H_{0}\left(g_{i}(t)\right) \geq \int_{g_{i}^{-1}(r)}^{t} \xi_{i}(s) d s \geq \int_{t_{1}}^{t} \xi(s) d s$ which means

$$
g_{i}(t) \leq H_{0}^{-1}\left(\int_{t_{1}}^{t} \xi(s) d s\right), \quad \forall t \geq t_{1}
$$

Also, it is evident, by the properties of $H, H_{0}$ and $H_{1}$, that

$$
H_{1}(t)=\int_{t}^{r} \frac{1}{s H^{\prime}(s)} d s \leq \int_{t}^{r} \frac{1}{H(s)} d s=H_{0}(t) \Longrightarrow H_{1}^{-1}(t) \leq H_{0}^{-1}(t) .
$$

This shows that (2.7) provides the best decay rates expected under the very general assumption (H1).

(2) Also, it is easy to notice that we can start the integration inside at zero where if $\bar{k}_{1}<k_{1}$ is chosen so that $\bar{k}_{1} \int_{0}^{2 t_{1}} \xi(s) d s=k_{1} \int_{t_{1}}^{2 t_{1}} \xi(s) d s$, then, as $H_{1}^{-1}$ is decreasing,

$$
E(t) \leq k_{2} H_{1}^{-1}\left(\bar{k}_{1} \int_{0}^{t} \xi(s) d s\right), \quad \forall t \geq 2 t_{1}
$$

(3) The above results allow relaxation functions which are not necessarily of exponential or polynomial decay and they are obtained under general hypotheses that allow to deal with a much larger class of functions $g_{1}, g_{2}$ that guarantee the uniform stability of (1.1) with an explicit formula for the decay rates of the energy. We give additional examples of application.

Examples. A- If $g_{1}(t)=g_{2}(t)=a \exp \left(-t^{q}\right)$, for $0<q<1$ and $a$ chosen so that $g_{i}$, for $i=1,2$, satisfy (2.1), then $g_{i}^{\prime}(t)=-H\left(g_{i}(t)\right)$ where $H(t)=\frac{q t}{[\ln (a / t)]^{\frac{1}{q}-1}}$. Since

$$
H^{\prime}(t)=\frac{(1-q)+q \ln \left(\frac{a}{t}\right)}{\left[\ln \left(\frac{a}{t}\right)\right]^{\frac{1}{q}}} \quad \text { and } \quad H^{\prime \prime}(t)=\frac{(1-q)\left[\ln \left(\frac{a}{t}\right)+\frac{1}{q}\right]}{\left[\ln \left(\frac{a}{t}\right)\right]^{\frac{1}{q}+1}}
$$


then the function $H$ satisfies hypothesis (H1) on the interval $(0, r]$ for any $0<r<a$. Therefore, we can use (2.7) to get

$$
\begin{aligned}
& H_{1}(t)=\int_{t}^{r} \frac{1}{s H^{\prime}(s)} d s=\int_{t}^{r} \frac{\left[\ln \frac{a}{s}\right]^{\frac{1}{q}}}{s\left[1-q+q \ln \frac{a}{s}\right]} d s=\int_{\ln \frac{a}{r}}^{\ln \frac{a}{t}} \frac{u^{\frac{1}{q}}}{1-q+q u} d u \\
& =\frac{1}{q} \int_{\ln \frac{a}{r}}^{\ln \frac{a}{t}} u^{\frac{1}{q}-1}\left[\frac{u}{\frac{1-q}{q}+u}\right] d u \leq \frac{1}{q} \int_{\ln \frac{a}{r}}^{\ln \frac{a}{t}} u^{\frac{1}{q}-1} d u \leq\left[\ln \frac{a}{t}\right]^{\frac{1}{q}} \\
& \Longrightarrow H_{1}^{-1}(t) \leq a \exp \left(-t^{q}\right) \Longrightarrow E(t) \leq a k_{2} \exp \left(-k_{3} t^{q}\right) .
\end{aligned}
$$

B- Considering the functions $g_{1}(t)=g_{2}(t)=\frac{a}{(t+e)[\ln (t+e)]^{p}}$ where $p>1$ and $a$ chosen so that (2.1) is satisfied. We can see that $g_{i}^{\prime}(t)=\frac{-a[\ln (t+e)+p]}{(t+e)^{2}[\ln (t+e)]^{p+1}}$ can be written as

$$
\begin{aligned}
g_{i}^{\prime}(t) & =\frac{-[\ln (t+e)+p]}{(t+e) \ln (t+e)} g(t) \\
\underset{\text { by }(2.8)-\text { case } 1}{\Longrightarrow} E(t) & \leq k e^{-k_{1} \int_{0}^{t} \frac{[\ln (t+e)+p]}{(t+e) \ln (t+e)} d s}=\frac{k}{\left((t+e)[\ln (t+e)]^{p}\right)^{k_{1}}}
\end{aligned}
$$

which is slower rate, as $k_{1} \leq 1$, than $g_{1}(t)$ and $g_{2}(t)$. But, it can also be written as

$$
\begin{aligned}
g_{i}^{\prime}(t) & =\frac{-[\ln (t+e)+p]}{a^{\frac{1}{p}}(t+e)^{1-\frac{1}{p}}}(g(t))^{1+\frac{1}{p}} \\
\text { by }(2.8)-\text { case } 2 & \left.E(t) \leq \bar{k}\left(1+\int_{0}^{t} \frac{\ln (t+e)+p}{a^{\frac{1}{p}}(t+e)^{1-\frac{1}{p}}}\right) d s\right)^{-p} \underset{\text { for large } t}{\leq} \frac{\bar{k}}{(t+e)[\ln (t+e)]^{p}}
\end{aligned}
$$

which is the same rate as the relaxation functions.

(4) The well-known Jensen's inequality will be of essential use in establishing our main result. If $F$ is a convex function on $[a, b], f: \Omega \rightarrow[a, b]$ and $h$ are integrable functions on $\Omega, h(x) \geq 0$, and $\int_{\Omega} h(x) d x=k>0$, then Jensen's inequality states that

$$
F\left[\frac{1}{k} \int_{\Omega} f(x) h(x) d x\right] \leq \frac{1}{k} \int_{\Omega} F[f(x)] h(x) d x .
$$

(5) We easily deduce, by (H1), that $\lim _{t \rightarrow+\infty} g_{i}(t)=0$. Hence, $t_{1}=\max \left\{g_{1}^{-1}(r), g_{2}^{-1}(r)\right\}$ is well-defined and, for $i=1,2$,

$$
g_{i}(t) \leq r, \quad \forall t \geq t_{1} .
$$

As $g_{i}$ and $\xi_{i}$ are positive nonincreasing continuous functions and $H$ is a positive continuous function, then, for all $t \in\left[0, t_{1}\right]$,

$$
\left\{\begin{array}{l}
0<g_{i}\left(t_{1}\right) \leq g_{i}(t) \leq g_{i}(0) \\
0<\xi_{i}\left(t_{1}\right) \leq \xi_{i}(t) \leq \xi_{i}(0)
\end{array} \Longrightarrow a \leq \xi_{i}(t) H\left(g_{i}(t)\right) \leq b\right.
$$


for some positive constants $a$ and $b$. Consequently, for all $t \in\left[0, t_{1}\right]$,

$$
g_{i}^{\prime}(t) \leq-\xi_{i}(t) H\left(g_{i}(t)\right) \leq-\frac{a}{M_{0}} M_{0} \leq-\frac{a}{M_{0}} g_{i}(t)
$$

(6) If different functions $H_{1}$ and $H_{2}$ have the properties mentioned in (H1) such that $g_{1}^{\prime}(t) \leq-\xi_{2}(t) H_{1}\left(g_{1}(t)\right)$ and $g_{2}^{\prime}(t) \leq-\xi_{2}(t) H_{2}\left(g_{2}(t)\right)$, then there is $r \leq \min \left\{r_{1}, r_{2}\right\}$ small enough so that, say, $H_{1}(t) \leq H_{2}(t)$ on the interval $(0, r]$. Thus, the function $H(t)=H_{1}(t)$ satisfies (H1) for both functions $g_{1}$ and $g_{2}, \forall t \geq t_{1}$.

(7) If $H$ is a strictly increasing and strictly convex $C^{2}$ function on $(0, r]$, with $H(0)=H^{\prime}(0)=0$, then it has an extension $\bar{H}$ which is strictly increasing and strictly convex $C^{2}$ function on $(0, \infty)$. For instance, if $H(r)=a, H^{\prime}(r)=b, H^{\prime \prime}(r)=c$, we can define $\bar{H}$, for $t>r$, by

$$
\bar{H}(t)=\frac{c}{2} t^{2}+(b-c r) t+\left(a+\frac{c}{2} r^{2}-b r\right) .
$$

\section{Proof of Theorem 2.1}

The existence is proved using Galerkin method. In order to do so, we take $\left\{w_{i}\right\}_{i=1}^{\infty}$ to be the eigenfunctions of the Laplacian operator subject to Dirichlet boundary conditions. Then $\left\{w_{i}\right\}_{i=1}^{\infty}$ is orthogonal basis of $H_{0}^{1}(\Omega)$ and of $H^{2}(\Omega) \cap H_{0}^{1}(\Omega)$ which is orthonormal in $L^{2}(\Omega)$. Let $V_{m}=\operatorname{span}\left\{w_{1}, w_{2}, \ldots, w_{m}\right\}$ and the projection of the initial data on the finite dimensional subspace $V_{m}$ is given by

$$
u_{0}^{m}=\sum_{j=1}^{m} a_{j} w_{j}, \quad v_{0}^{m}=\sum_{j=1}^{m} b_{j} w_{j}, \quad u_{1}^{m}=\sum_{j=1}^{m} c_{j} w_{j}, \quad v_{1}^{m}=\sum_{j=1}^{m} d_{j} w_{j}
$$

where, $\left(u_{0}^{m}, v_{0}^{m}\right) \rightarrow\left(u_{0}, v_{0}\right)$ in $\left(H^{2}(\Omega) \cap H_{0}^{1}(\Omega)\right)^{2}$ and $\left(u_{1}^{m}, v_{1}^{m}\right) \rightarrow\left(u_{1}, v_{1}\right)$ in $\left(H_{0}^{1}(\Omega)\right)^{2}$ as $m \rightarrow \infty$. We search the approximate solutions

$$
u^{m}(x, t)=\sum_{j=1}^{m} h_{j, m}(t) w_{j}(x), \quad v^{m}(x, t)=\sum_{j=1}^{m} k_{j, m}(t) w_{j}(x)
$$

of the approximate problem in $V_{m}$

$$
\left\{\begin{array}{c}
\int_{\Omega}\left(u_{t t}^{m} w+\nabla u^{m} \cdot \nabla w-\int_{0}^{t} g_{1}(t-\tau) \nabla u^{m}(\tau) \cdot \nabla w d \tau+f_{1}\left(u^{m}, v^{m}\right) w\right) d x=0 \\
\int_{\Omega}\left(v_{t t}^{m} w+\nabla v^{m} \cdot \nabla w-\int_{0}^{t} g_{2}(t-\tau) \nabla v^{m}(\tau) \cdot \nabla w d \tau+f_{2}\left(u^{m}, v^{m}\right) w\right) d x=0 \\
u^{m}(0)=u_{0}^{m}, \quad u_{t}^{m}(0)=u_{1}^{m}, \quad v^{m}(0)=v_{0}^{m}, \quad v_{t}^{m}(0)=v_{1}^{m} .
\end{array}\right.
$$

This system leads to a system of ODE for uknown functions $h_{j, m}(t), k_{j, m}(t)$. Based on standard existence theory for ODE, one can conclude the existence of a solution $\left(u^{m}, v^{m}\right)$ of (3.1) on a maximal time interval $\left[0, t_{m}\right)$, for each $m \in \mathbb{N}$. The a priori estimate that follows implies that in fact $t_{m}=+\infty$.

- (A priori estimate 1): In (3.1), let $w=u_{t}^{m}$ in the first equation and $w=v_{t}^{m}$ in the second equation, add the resulting equations, and integrate by parts to obtain

$$
\frac{d}{d t} E^{m}(t)=\frac{1}{2}\left(g_{1}^{\prime} \circ \nabla u^{m}\right)-\frac{1}{2} g_{1}(t)\left\|\nabla u^{m}\right\|_{2}^{2}+\frac{1}{2}\left(g_{2}^{\prime} \circ \nabla v^{m}\right)-\frac{1}{2} g_{2}(t)\left\|\nabla v^{m}\right\|_{2}^{2} .
$$

This means, using (H1), that, for some positive constant $C$ independent of $t$ and $m$,

$$
E^{m}(t) \leq E^{m}(0) \leq C \text {. }
$$


- (A priori estimate 2): In (3.1), let $w=-\Delta u_{t}^{m}$ in the first equation and $w=-\Delta v_{t}^{m}$ in the second equation, add the resulting equations, integrate by parts, and use (H1) to obtain

$$
\begin{aligned}
& \frac{1}{2} \frac{d}{d t}\left[\begin{array}{c}
\left\|\nabla u_{t}^{m}\right\|_{2}^{2}+\left(1-\int_{0}^{t} g_{1}(s) d s\right)\left\|\Delta u^{m}\right\|_{2}^{2}+\left\|\nabla v_{t}^{m}\right\|_{2}^{2} \\
+\left(1-\int_{0}^{t} g_{2}(s) d s\right)\left\|\Delta v^{m}\right\|_{2}^{2}+g_{1} \circ \Delta u^{m}+g_{2} \circ \Delta v^{m}
\end{array}\right] \\
= & \frac{1}{2}\left(g_{1}^{\prime} \circ \Delta u^{m}\right)-\frac{1}{2} g_{1}(t)\left\|\Delta u^{m}\right\|_{2}^{2}+\frac{1}{2}\left(g_{2}^{\prime} \circ \Delta v^{m}\right)-\frac{1}{2} g_{2}(t)\left\|\Delta v^{m}\right\|_{2}^{2} \\
& +\int_{\Omega}\left(f_{1}\left(u^{m}, v^{m}\right) \Delta u_{t}^{m}+f_{2}\left(u^{m}, v^{m}\right) \Delta v_{t}^{m}\right) d x \\
\leq & \int_{\Omega}\left(f_{1}\left(u^{m}, v^{m}\right) \Delta u_{t}^{m}+f_{2}\left(u^{m}, v^{m}\right) \Delta v_{t}^{m}\right) d x
\end{aligned}
$$

Then, integrating over $(0, t)$ yields

$$
\begin{aligned}
& \frac{1}{2}\left[\begin{array}{c}
\left\|\nabla u_{t}^{m}\right\|_{2}^{2}+\left(1-\int_{0}^{t} g_{1}(s) d s\right)\left\|\Delta u^{m}\right\|_{2}^{2}+\left\|\nabla v_{t}^{m}\right\|_{2}^{2} \\
+\left(1-\int_{0}^{t} g_{2}(s) d s\right)\left\|\Delta v^{m}\right\|_{2}^{2}+g_{1} \circ \Delta u^{m}+g_{2} \circ \Delta v^{m}
\end{array}\right] \\
\leq & \frac{1}{2}\left[\left\|\nabla u_{1}^{m}\right\|_{2}^{2}+\left\|\Delta u_{0}^{m}\right\|_{2}^{2}+\left\|\nabla v_{1}^{m}\right\|_{2}^{2}+\left\|\Delta v_{0}^{m}\right\|_{2}^{2}\right] \\
+ & \int_{\Omega}\left(\begin{array}{c}
f_{1}\left(u^{m}, v^{m}\right) \Delta u^{m}-f_{1}\left(u_{0}^{m}, v_{0}^{m}\right) \Delta u_{0}^{m} \\
+f_{2}\left(u^{m}, v^{m}\right) \Delta v^{m}-f_{2}\left(u_{0}^{m}, v_{0}^{m}\right) \Delta v_{0}^{m}
\end{array}\right) d x \\
& -\int_{0}^{t} \int_{\Omega}\left(\begin{array}{c}
\frac{\partial f_{1}}{\partial u}\left(u^{m}, v^{m}\right) u_{t}^{m} \Delta u^{m}+\frac{\partial f_{1}}{\partial v}\left(u^{m}, v^{m}\right) v_{t}^{m} \Delta u^{m} \\
+\frac{\partial f_{2}}{\partial u}\left(u^{m}, v^{m}\right) u_{t}^{m} \Delta v^{m}+\frac{\partial f_{2}}{\partial v}\left(u^{m}, v^{m}\right) v_{t}^{m} \Delta v^{m}
\end{array}\right) d x d s
\end{aligned}
$$

To estimate the terms in the right hand side of (3.3), we use (2.4), Young's inequality, and (2.6) and take (3.2) into account to get

$$
\begin{aligned}
& \int_{\Omega} f_{1}\left(u^{m}, v^{m}\right) \Delta u^{m} d x \leq k \int_{\Omega}\left(\left|u^{m}\right|+\left|v^{m}\right|+\left|u^{m}\right|^{\beta_{11}}+\left|v^{m}\right|^{\beta_{12}}\right)\left|\Delta u^{m}\right| d x \\
& \leq \delta\left\|\Delta u^{m}\right\|_{2}^{2}+\frac{c}{\delta} \int_{\Omega}\left(\left|u^{m}\right|^{2}+\left|v^{m}\right|^{2}+\left|u^{m}\right|^{2 \beta_{11}}+\left|v^{m}\right|^{2 \beta_{12}}\right) d x \\
& \leq \delta\left\|\Delta u^{m}\right\|_{2}^{2}+\frac{c}{\delta}\left(\left\|\nabla u^{m}\right\|_{2}^{2}+\left\|\nabla v^{m}\right\|_{2}^{2}+\left\|\nabla u^{m}\right\|_{2}^{2 \beta_{11}}+\left\|\nabla v^{m}\right\|_{2}^{2 \beta_{12}}\right) \\
& \leq \delta\left\|\Delta u^{m}\right\|_{2}^{2}+\frac{c}{\delta} .
\end{aligned}
$$

Now, we estimate $J:=-\int_{\Omega} \frac{\partial f_{1}}{\partial u}\left(u^{m}, v^{m}\right) u_{t}^{m} \Delta u^{m} d x$ as follows. First, we observe that $\frac{\beta_{1 j}-1}{2 \beta_{1 j}}+\frac{1}{2 \beta_{1 j}}+\frac{1}{2}=$ 1 and use (H2) and the generalized Hölder's inequality to infer

$$
\begin{aligned}
|J| & \leq d \int_{\Omega}\left(1+\left|u^{m}\right|^{\beta_{11}-1}+\left|v^{m}\right|^{\beta_{12}-1}\right)\left|u_{t}^{m}\right|\left|\Delta u^{m}\right| d x \\
& \leq d\left(\left\|u_{t}^{m}\right\|_{2}+\left\|u^{m}\right\|_{2 \beta_{11}}^{\beta_{11}-1}\left\|u_{t}^{m}\right\|_{2 \beta_{11}}+\left\|v^{m}\right\|_{2 \beta_{12}}^{\beta_{12}-1}\left\|u_{t}^{m}\right\|_{2 \beta_{12}}\right)\left\|\Delta u^{m}\right\|_{2}
\end{aligned}
$$


Then, by (2.6), (3.2), and Young's inequality, we arrive at

$$
\begin{aligned}
|J| & \leq c\left(1+\left\|\nabla u^{m}\right\|_{2}^{\beta_{11}-1}+\left\|\nabla v^{m}\right\|_{2}^{\beta_{12}-1}\right)\left\|\nabla u_{t}^{m}\right\|_{2}\left\|\Delta u^{m}\right\|_{2} \\
& \leq c\left\|\nabla u_{t}^{m}\right\|_{2}\left\|\Delta u^{m}\right\|_{2} \leq c\left\|\nabla u_{t}^{m}\right\|_{2}^{2}+c\left\|\Delta u^{m}\right\|_{2}^{2} .
\end{aligned}
$$

Since the other terms in (3.3) can be similarly treated and the norms of the initial data are uniformly bounded, we combine (3.3-3.5), use (H1), and take $\delta$ small enough to end up with

$$
\begin{gathered}
\left\|\nabla u_{t}^{m}\right\|_{2}^{2}+\left\|\Delta u^{m}\right\|_{2}^{2}+\left\|\nabla v_{t}^{m}\right\|_{2}^{2}+\left\|\Delta v^{m}\right\|_{2}^{2} \\
\leq c+c \int_{0}^{t}\left(\left\|\nabla u_{t}^{m}\right\|_{2}^{2}+\left\|\Delta u^{m}\right\|_{2}^{2}+\left\|\nabla v_{t}^{m}\right\|_{2}^{2}+\left\|\Delta v^{m}\right\|_{2}^{2}\right) d s .
\end{gathered}
$$

Using Gronwall's inequality, this implies that

$$
\left\|\nabla u_{t}^{m}\right\|_{2}^{2}+\left\|\Delta u^{m}\right\|_{2}^{2}+\left\|\nabla v_{t}^{m}\right\|_{2}^{2}+\left\|\Delta v^{m}\right\|_{2}^{2} \leq C, \quad \forall t \in[0, T] \text { and } m \in \mathbb{N} .
$$

- (A priori estimate 3): In (3.1), let $w=u_{t t}^{m}$ in the first equation and $w=v_{t t}^{m}$ in the second equation. Then, by exploiting the previous estimates and using similar arguments, we find

$$
\left\|u_{t t}^{m}\right\|_{2}^{2}+\left\|v_{t t}^{m}\right\|_{2}^{2} \leq C, \quad \forall t \in[0, T] \text { and } m \in \mathbb{N} .
$$

From (3.2), (3.6), and (3.7), we conclude that

$u^{m}, v^{m}$ are uniformly bounded in $L^{\infty}\left(0, T ; H^{2}(\Omega) \cap H_{0}^{1}(\Omega)\right)$,

$u_{t}^{m}, v_{t}^{m}$ are uniformly bounded in $L^{\infty}\left(0, T ; H_{0}^{1}(\Omega)\right)$,

$u_{t t}^{m}, v_{t t}^{m}$ are uniformly bounded in $L^{\infty}\left(0, T ; L^{2}(\Omega)\right)$,

which implies that there exist subsequences of $u^{m}, v^{m}$, which we still denote in the same way, such that

$$
\begin{aligned}
& u^{m} \stackrel{*}{\rightarrow} u \text { and } v^{m} \stackrel{*}{\rightarrow} v \quad \text { in } L^{\infty}\left(0, T ; H^{2}(\Omega) \cap H_{0}^{1}(\Omega)\right), \\
& u_{t}^{m} \stackrel{*}{\rightarrow} u_{t} \text { and } v_{t}^{m} \stackrel{*}{\rightarrow} v_{t} \quad \text { in } L^{\infty}\left(0, T ; H_{0}^{1}(\Omega)\right), \\
& u_{t t}^{m} \stackrel{*}{\rightarrow} u_{t t} \text { and } v_{t t}^{m} \stackrel{*}{*} v_{t t} \quad \text { in } L^{\infty}\left(0, T ; L^{2}(\Omega)\right) .
\end{aligned}
$$

In particular, making use of Aubin-Lions Theorem, we find, up to a subsequence, that

$$
u^{m} \rightarrow u \text { and } v^{m} \rightarrow v \quad \text { strongly in } L^{2}\left(0, T ; L^{2}(\Omega)\right) .
$$

Then,

$$
u^{m} \rightarrow u \text { and } v^{m} \rightarrow v \quad \text { a.e in }(0, T) \times \Omega
$$

and therefore, from $(\mathrm{H} 2)$,

$$
f_{i}\left(u^{m}, v^{m}\right) \rightarrow f_{i}(u, v) \quad \text { a.e in }(0, T) \times \Omega, \text { for } i=1,2 .
$$

Also, as $u^{m}$ and $v^{m}$ are bounded in $L^{\infty}\left(0, T ; L^{2}(\Omega)\right)$, then the use of (2.4) and (2.6) gives that $f_{i}\left(u^{m}, v^{m}\right)$ is bounded in $L^{\infty}\left(0, T ; L^{2}(\Omega)\right)$. From this and (3.9), we can deduce that

$$
f_{i}\left(u^{m}, v^{m}\right) \rightarrow f_{i}(u, v) \quad \text { in } L^{2}\left(0, T ; L^{2}(\Omega)\right), \text { for } i=1,2 .
$$


Regarding the initial conditions, we can also use (3.8) in standard way to verify that

$$
u(0)=u_{0}, \quad u_{t}(0)=u_{1}, \quad v(0)=v_{0}, \quad v_{t}(0)=v_{1}
$$

Combining the results obtained above, we can pass to the limit and conclude that $(u, v)$ is a strong solution of system (1.1). For uniqueness, let us assume that $\left(u_{1}, v_{1}\right),\left(u_{2}, v_{2}\right)$ are two strong solutions of (1.1). Then, $(z, q)=\left(u_{1}-u_{2}, v_{1}-v_{2}\right)$ satisfies, for all $w \in H_{0}^{1}(\Omega)$,

$$
\left\{\begin{array}{l}
\int_{\Omega}\left(z_{t t} w+\nabla z . \nabla w-\int_{0}^{t} g_{1}(t-\tau) \nabla z(\tau) . \nabla w d \tau\right) d x=\int_{\Omega}\left(f_{1}\left(u_{2}, v_{2}\right)-f_{1}\left(u_{1}, v_{1}\right)\right) w d x \\
\int_{\Omega}\left(q_{t t} w+\nabla q . \nabla w-\int_{0}^{t} g_{2}(t-\tau) \nabla q(\tau) . \nabla w d \tau\right) d x=\int_{\Omega}\left(f_{2}\left(u_{2}, v_{2}\right)-f_{2}\left(u_{1}, v_{1}\right)\right) w d x .
\end{array}\right.
$$

Substituting $w=z_{t}$ in the first equation and $w=q_{t}$ in the second equation, adding the resulting equations, integrating by parts, and using (H1) yield

$$
\begin{aligned}
& \frac{1}{2} \frac{d}{d t}\left[\begin{array}{c}
\left\|z_{t}\right\|_{2}^{2}+\left(1-\int_{0}^{t} g_{1}(s) d s\right)\|\nabla z\|_{2}^{2}+\left\|q_{t}\right\|_{2}^{2} \\
+\left(1-\int_{0}^{t} g_{2}(s) d s\right)\|\nabla q\|_{2}^{2}+g_{1} \circ \nabla z+g_{2} \circ \nabla q
\end{array}\right] \\
\leq & \int_{\Omega}\left(f_{1}\left(u_{2}, v_{2}\right)-f_{1}\left(u_{1}, v_{1}\right)\right) z_{t} d x+\int_{\Omega}\left(f_{2}\left(u_{2}, v_{2}\right)-f_{2}\left(u_{1}, v_{1}\right)\right) q_{t} d x .
\end{aligned}
$$

Making use of (2.5) and following similar arguments used to obtain (3.5), we find

$$
\begin{aligned}
& \int_{\Omega}\left(f_{1}\left(u_{2}, v_{2}\right)-f_{1}\left(u_{1}, v_{1}\right)\right) z_{t} d x+\int_{\Omega}\left(f_{2}\left(u_{2}, v_{2}\right)-f_{2}\left(u_{1}, v_{1}\right)\right) q_{t} d x \\
\leq & k \int_{\Omega}\left(1+\left|u_{1}\right|^{\beta_{11}-1}+\left|u_{2}\right|^{\beta_{11}-1}+\left|v_{1}\right|^{\beta_{12}-1}+\left|v_{2}\right|^{\beta_{12}-1}\right)(|z|+|q|)\left|z_{t}\right| d x \\
& +k \int_{\Omega}\left(1+\left|u_{1}\right|^{\beta_{21}-1}+\left|u_{2}\right|^{\beta_{21}-1}+\left|v_{1}\right|^{\beta_{22}-1}+\left|v_{2}\right|^{\beta_{22}-1}\right)(|z|+|q|)\left|q_{t}\right| d x \\
\leq & c\left(\left\|z_{t}\right\|_{2}^{2}+\|\nabla z\|_{2}^{2}+\left\|q_{t}\right\|_{2}^{2}+\|\nabla q\|_{2}^{2}\right) .
\end{aligned}
$$

Combining (3.10) and (3.11), integrating over $(0, t)$, and using Gronwall's Lemma, then we deduce that

$$
\left\|z_{t}\right\|_{2}^{2}+\|\nabla z\|_{2}^{2}+\left\|q_{t}\right\|_{2}^{2}+\|\nabla q\|_{2}^{2}=0
$$

which means that $\left(u_{1}, v_{1}\right)=\left(u_{2}, v_{2}\right)$. This completes the proof.

Now, if $\left(u_{0}, u_{1}\right),\left(v_{0}, v_{1}\right) \in H_{0}^{1}(\Omega) \times L^{2}(\Omega)$ and considering standard density arguments, we can prove that problem (1.1) has a unique weak solution

$$
u, v \in C\left(\mathbb{R}_{+} ; H_{0}^{1}(\Omega)\right) \cap C^{1}\left(\mathbb{R}_{+} ; L^{2}(\Omega)\right) .
$$

\section{Technical lemmas}

In this section, we establish several lemmas needed to prove Theorem 2.2.

Lemma 4.1. Let $(u, v)$ be the solution of (1.1). Then the energy functional satisfies

$$
E^{\prime}(t)=\frac{1}{2}\left(g_{1}^{\prime} \circ \nabla u\right)-\frac{1}{2} g_{1}(t) \int_{\Omega}|\nabla u|^{2} d x+\frac{1}{2}\left(g_{2}^{\prime} \circ \nabla v\right)-\frac{1}{2} g_{2}(t) \int_{\Omega}|\nabla v|^{2} d x \leq 0 .
$$


Proof. By multiplying the first equation in (1.1) by $u_{t}$ and the second by $v_{t}$, integrating over $\Omega$, using integration by parts, hypotheses $(\mathrm{H} 1)$ and $(\mathrm{H} 2)$, and some manipulations, we obtain (4.1) for any regular solution. This equality remains valid for weak solutions by a simple density argument.

Now we are going to construct a Lyapunov functional $\mathcal{L}$ equivalent to $E$, with which we can show the desired result.

Lemma 4.2. Under the assumptions (H1) and (H2), the functional I defined by

$$
I(t):=\int_{\Omega} u u_{t} d x+\int_{\Omega} v v_{t} d x
$$

satisfies, along the solution, with $l=\min \left\{l_{1}, l_{2}\right\}$, the estimate

$$
\begin{aligned}
I^{\prime}(t) \leq & -\frac{l}{2} \int_{\Omega}\left(|\nabla u|^{2}+|\nabla v|^{2}\right) d x+\int_{\Omega}\left(u_{t}^{2}+v_{t}^{2}\right) d x+c C_{\alpha_{1}}\left(h_{1} \circ \nabla u\right)(t) \\
& +c C_{\alpha_{2}}\left(h_{2} \circ \nabla v\right)(t)-\int_{\Omega} F(u, v) d x .
\end{aligned}
$$

for any $0<\alpha_{i}<1(i=1,2)$, where

$$
C_{\alpha_{i}}=\int_{0}^{\infty} \frac{g_{i}^{2}(s)}{\alpha_{i} g_{i}(s)-g_{i}^{\prime}(s)} d s \quad \text { and } \quad h_{i}(t)=\alpha_{i} g_{i}(t)-g_{i}^{\prime}(t)
$$

Proof. Direct computations, using (1.1), (H1-H2), and Young's inequality, yield

$$
\begin{aligned}
& I^{\prime}(t)= \int_{\Omega} u_{t}^{2} d x-\int_{\Omega}|\nabla u|^{2} d x+\int_{\Omega} \nabla u \cdot \int_{0}^{t} g_{1}(t-\tau) \nabla u(\tau) d \tau d x \\
&+\int_{\Omega} v_{t}^{2} d x-\int_{\Omega}|\nabla v|^{2} d x+\int_{\Omega} \nabla v \cdot \int_{0}^{t} g_{2}(t-\tau) \nabla v(\tau) d \tau d x \\
&-\int_{\Omega}\left[u f_{1}(u, v)+v f_{2}(u, v)\right] d x \\
&\left.=\int_{\Omega} u_{t}^{2} d x-\int_{0} 1-\int_{0}^{t} g_{1}(s) d s\right) \int_{\Omega}|\nabla u|^{2} d x+\int_{\Omega} \nabla u \cdot \int_{0}^{t} g_{1}(t-\tau)(\nabla u(\tau)-\nabla u(t)) d \tau d x \\
&+\int_{\Omega} v_{t}^{2} d x-\left(\begin{array}{c}
\left.1-\int_{0} g_{1}(s) d s\right) \\
-\int_{\Omega}\left[u f_{1}(u, v)+v f_{2}(u, v)\right] d x
\end{array} \mid \int_{\Omega} g_{2}(t-\tau)(\nabla v(\tau)-\nabla v(t)) d \tau d x\right.
\end{aligned}
$$




$$
\begin{aligned}
& \leq \int_{\Omega} u_{t}^{2} d x-l \int_{\Omega}|\nabla u|^{2} d x+\delta \int_{\Omega}|\nabla u|^{2} d x+\frac{1}{4 \delta} \int_{\Omega}\left(\int_{0}^{t} g_{1}(t-\tau)|\nabla u(\tau)-\nabla u(t)| d \tau\right)^{2} d x \\
& +\int_{\Omega} v_{t}^{2} d x-l \int_{\Omega}|\nabla v|^{2} d x+\delta \int_{\Omega}|\nabla v|^{2} d x+\frac{1}{4 \delta} \int_{\Omega}\left(\int_{0}^{t} g_{2}(t-\tau)|\nabla v(\tau)-\nabla v(t)| d \tau\right)^{2} d x \\
& -\int_{\Omega} F(u, v) d x
\end{aligned}
$$

for any $\delta>0$. Now, the use of Cauchy-Schwarz inequality gives

$$
\begin{aligned}
& \int_{\Omega}\left(\int_{0}^{t} g_{1}(t-\tau)|\nabla u(\tau)-\nabla u(t)| d \tau\right)^{2} d x \\
= & \int_{\Omega}\left(\int_{0}^{t} \frac{g_{1}(t-\tau)}{\sqrt{\alpha_{1} g_{1}(t-\tau)-g_{1}^{\prime}(t-\tau)}} \sqrt{\alpha_{1} g_{1}(t-\tau)-g_{1}^{\prime}(t-\tau)}|\nabla u(\tau)-\nabla u(t)| d s\right)^{2} d x \\
\leq & \left.\int_{0}^{t} \frac{g_{1}^{2}(s)}{\alpha_{1} g_{1}(s)-g_{1}^{\prime}(s)} d s\right) \int_{\Omega} \int_{0}^{t}\left[\alpha_{1} g_{1}(t-\tau)-g_{1}^{\prime}(t-\tau)\right]|\nabla u(\tau)-\nabla u(t)|^{2} d \tau d x \\
\leq & C_{\alpha_{1}}\left(h_{1} \circ \nabla u\right)(t) .
\end{aligned}
$$

Similar calculations also yield

$$
\int_{\Omega}\left(\int_{0}^{t} g_{2}(t-\tau)|\nabla v(\tau)-\nabla v(t)| d \tau\right)^{2} d x \leq C_{\alpha_{2}}\left(h_{2} \circ \nabla v\right)(t) .
$$

Combining (4.4)-(4.6) and choosing $\delta$ small enough give (4.2).

Lemma 4.3. Under the assumptions (H1) and (H2), the functional $K$ defined by

$$
K(t)=K_{1}(t)+K_{2}(t)
$$

with

$$
\begin{aligned}
& K_{1}(t):=-\int_{\Omega} u_{t} \int_{0}^{t} g_{1}(t-\tau)(u(t)-u(\tau)) d \tau d x \\
& K_{2}(t):=-\int_{\Omega} v_{t} \int_{0}^{t} g_{2}(t-\tau)(v(t)-v(\tau)) d \tau d x
\end{aligned}
$$


satisfies, for any $0<\delta<1$ and all $t \geq t_{1}$, the estimate

$$
\begin{aligned}
K^{\prime}(t) \leq & -\left(g_{0}-\delta\right) \int_{\Omega}\left(u_{t}^{2}+v_{t}^{2}\right) d x+\delta c \int_{\Omega}\left(|\nabla u|^{2}+|\nabla v|^{2}\right) d x \\
& +\frac{c\left[C_{\alpha_{1}}+1\right]}{\delta}\left(h_{1} \circ \nabla u\right)(t)+\frac{c\left[C_{\alpha_{2}}+1\right]}{\delta}\left(h_{2} \circ \nabla v\right)(t)
\end{aligned}
$$

where $g_{0}=\min \left\{\int_{0}^{t_{1}} g_{1}(s) d s, \int_{0}^{t_{1}} g_{2}(s) d s\right\}$.

Proof. By exploiting equations (1.1) and integrating by parts, we have

$$
\begin{aligned}
& K_{1}^{\prime}(t)=\left(1-\int_{0}^{t} g_{1}(s) d s\right) \int_{\Omega} \nabla u \cdot \int_{0}^{t} g_{1}(t-\tau)(\nabla u(t)-\nabla u(\tau)) d \tau d x \\
& +\int_{\Omega}\left(\int_{0}^{t} g_{1}(t-\tau)|\nabla u(t)-\nabla u(\tau)| d \tau\right)^{2} d x+\int_{\Omega} f_{1}(u, v) \int_{0}^{t} g_{1}(t-\tau)(u(t)-u(\tau)) d \tau d x \\
& -\int_{\Omega} u_{t} \int_{0}^{t} g_{1}^{\prime}(t-\tau)(u(t)-u(\tau)) d \tau d x-\left(\int_{0}^{t} g_{1}(s) d s\right) \int_{\Omega} u_{t}^{2} d x .
\end{aligned}
$$

Using Young's inequality and similar calculations in (4.5), we obtain

$$
\left(1-\int_{0}^{t} g_{1}(s) d s\right) \int_{\Omega} \nabla u \cdot \int_{0}^{t} g_{1}(t-\tau)(\nabla u(t)-\nabla u(\tau)) d \tau d x \leq \delta \int_{\Omega}|\nabla u|^{2} d x+\frac{c C_{\alpha_{1}}}{\delta}\left(h_{1} \circ \nabla u\right)(t)
$$

and

$$
\begin{aligned}
& -\int_{\Omega} u_{t} \int_{0}^{t} g_{1}^{\prime}(t-\tau)(u(t)-u(\tau)) d \tau d x \\
= & \int_{\Omega} u_{t} \int_{0}^{t} h_{1}(t-\tau)(u(t)-u(\tau)) d \tau d x-\int_{\Omega} u_{t} \int_{0}^{t} \alpha_{1} g_{1}(t-\tau)(u(t)-u(\tau)) d \tau d x \\
\leq & \frac{c}{\delta} \int_{\Omega}\left(\int_{0}^{t} \sqrt{h_{1}(t-\tau)} \sqrt{h_{1}(t-\tau)}|u(t)-u(\tau)| d \tau\right)^{2} d x \\
& +\delta \int_{\Omega} u_{t}^{2} d x+\frac{c \alpha_{1}^{2}}{\delta} \int_{\Omega}\left(\int_{0}^{t} g_{1}(t-\tau)|u(t)-u(\tau)| d \tau\right)^{2} d x \\
\leq & \delta \int_{\Omega} u_{t}^{2} d x+\frac{c\left(\int_{0}^{t} h_{1}(s) d s\right)}{\delta}\left(h_{1} \circ u\right)+\frac{c \alpha_{1}^{2} C_{\alpha_{1}}}{\delta}\left(h_{1} \circ u\right) \\
\leq & \delta \int_{\Omega} u_{t}^{2} d x+\frac{c}{\delta}\left(h_{1} \circ \nabla u\right)+\frac{c C_{\alpha_{1}}}{\delta}\left(h_{1} \circ \nabla u\right) .
\end{aligned}
$$


To estimate the third term in the right hand side of (4.8), we use (2.4), (2.6) and the fact that $\|\nabla u\|_{2}^{2}+$ $\|\nabla v\|_{2}^{2} \leq 2 E(t) \leq 2 E(0)$ to get

$$
\begin{aligned}
& \int_{\Omega} f_{1}(u, v) \int_{0}^{t} g_{1}(t-\tau)(u(t)-u(\tau)) d \tau d x \\
\leq & \delta c \int_{\Omega}\left(|u|^{2}+|v|^{2}+|u|^{2 \beta_{11}}+|v|^{2 \beta_{12}}\right) d x+\frac{c}{\delta} \int_{\Omega}\left(\int_{0}^{t} g_{1}(t-\tau)(u(t)-u(\tau)) d \tau\right)^{2} d x \\
\leq & \delta c\left(\|\nabla u\|_{2}^{2}+\|\nabla v\|_{2}^{2}+\|\nabla u\|_{2}^{2 \beta_{11}}+\|\nabla v\|_{2}^{2 \beta_{12}}\right)+\frac{c}{\delta} C_{\alpha_{1}}\left(h_{1} \circ \nabla u\right)(t) \\
= & \delta c\left(\|\nabla u\|_{2}^{2}+\|\nabla v\|_{2}^{2}+\|\nabla u\|_{2}^{2\left(\beta_{11}-1\right)}\|\nabla u\|_{2}^{2}+\|\nabla v\|_{2}^{2\left(\beta_{12}-1\right)}\|\nabla v\|_{2}^{2}\right)+\frac{c}{\delta}\left(h_{1} \circ \nabla u\right)(t) \\
\leq & \delta c\left(\|\nabla u\|_{2}^{2}+\|\nabla v\|_{2}^{2}+[2 E(0)]^{\left(\beta_{11}-1\right)}\|\nabla u\|_{2}^{2}+[2 E(0)]^{\left(\beta_{12}-1\right)}\|\nabla v\|_{2}^{2}\right)+\frac{c}{\delta}\left(h_{1} \circ \nabla u\right)(t) \\
\leq & \delta c\|\nabla u\|_{2}^{2}+\delta c\|\nabla v\|_{2}^{2}+\frac{c}{\delta}\left(h_{1} \circ \nabla u\right)(t) .
\end{aligned}
$$

By combining (4.8)-(4.11), we obtain

$$
\begin{aligned}
K_{1}^{\prime}(t) \leq & -\left(\int_{0}^{t} g_{1}(s) d s-\delta\right) \int_{\Omega} u_{t}^{2} d x+\delta c \int_{\Omega}|\nabla u|^{2} d x \\
& +\frac{c\left[C_{\alpha_{1}}+1\right]}{\delta}\left(h_{1} \circ \nabla u\right)(t)+\delta c \int_{\Omega}|\nabla v|^{2} d x .
\end{aligned}
$$

Since $K_{2}$ can be dealt with similarly, (4.7) is established.

Next, we use the functional

$$
M(t)=\int_{\Omega} \int_{0}^{t} f_{1}(t-\tau)|\nabla u(\tau)|^{2} d \tau d x+\int_{\Omega} \int_{0}^{t} f_{2}(t-\tau)|\nabla v(\tau)|^{2} d \tau d x
$$

where $f_{i}(t)=\int_{t}^{\infty} g_{i}(s) d s$.

Lemma 4.4. Assume that (H1) and (H2) hold. The functional $M$ satisfies, along the solution of (1.1), the estimate

$$
M^{\prime}(t) \leq-\frac{1}{2}\left(g_{1} \circ \nabla u\right)(t)-\frac{1}{2}\left(g_{2} \circ \nabla v\right)(t)+3(1-l) \int_{\Omega}\left(|\nabla u(t)|^{2}+|\nabla v(t)|^{2}\right) d x .
$$

Proof. By Young's inequality and the fact $f_{i}^{\prime}(t)=-g_{i}(t)$, we see that

$$
\begin{aligned}
M^{\prime}(t)= & f_{1}(0) \int_{\Omega}|\nabla u(t)|^{2} d x-\int_{\Omega} \int_{0}^{t} g_{1}(t-\tau)|\nabla u(\tau)|^{2} d \tau d x \\
& +f_{2}(0) \int_{\Omega}|\nabla v(t)|^{2} d x-\int_{\Omega} \int_{0}^{t} g_{2}(t-\tau)|\nabla v(\tau)|^{2} d \tau d x
\end{aligned}
$$




$$
\begin{aligned}
= & -\int_{\Omega} \int_{0}^{t} g_{1}(t-\tau)|\nabla u(\tau)-\nabla u(t)|^{2} d \tau d x \\
& -2 \int_{\Omega} \nabla u(t) \cdot \int_{0}^{t} g_{1}(t-\tau)(\nabla u(\tau)-\nabla u(t)) d \tau d x+f_{1}(t) \int_{\Omega}|\nabla u(t)|^{2} d x \\
& -\int_{\Omega} \int_{0}^{t} g_{2}(t-\tau)|\nabla v(\tau)-\nabla v(t)|^{2} d \tau d x \\
& -2 \int_{\Omega} \nabla v(t) \cdot \int_{0}^{t} g_{2}(t-\tau)(\nabla v(\tau)-\nabla v(t)) d \tau d x+f_{2}(t) \int_{\Omega}|\nabla v(t)|^{2} d x .
\end{aligned}
$$

But

$$
\begin{aligned}
& -2 \int_{\Omega} \nabla w(t) \cdot \int_{0}^{t} g_{i}(t-\tau)(\nabla w(\tau)-\nabla w(t)) d \tau d x \\
\leq & 2\left(1-l_{i}\right) \int_{\Omega}|\nabla w(t)|^{2} d x+\frac{\int_{0}^{t} g_{i}(s) d s}{2\left(1-l_{i}\right)} \int_{\Omega} \int_{0}^{t} g_{i}(t-\tau)|\nabla w(\tau)-\nabla w(t)|^{2} d \tau d x .
\end{aligned}
$$

Then, as $f_{i}(t) \leq f_{i}(0)=\left(1-l_{i}\right)$ and $\int_{0}^{t} g_{i}(s) d s \leq\left(1-l_{i}\right)$, we get (4.13).

\section{Lemma 4.5. The functional $\mathcal{L}$ defined by}

$$
\mathcal{L}(t):=N E(t)+N_{1} I(t)+N_{2} K(t)
$$

satisfies, for suitable choice of $N, N_{1}, N_{2}>0$, that

$$
\begin{gathered}
\mathcal{L}^{\prime}(t) \leq-4(1-l) \int_{\Omega}\left(|\nabla u|^{2}+|\nabla v|^{2}\right) d x-\int_{\Omega}\left(u_{t}^{2}+v_{t}^{2}\right) d x-\int_{\Omega} F(u, v) d x \\
+\frac{1}{4}\left(g_{1} \circ \nabla u\right)(t)+\frac{1}{4}\left(g_{2} \circ \nabla v\right)(t), \quad \forall t \geq t_{1}
\end{gathered}
$$

and

$$
\mathcal{L}(t) \sim E(t)
$$

Proof. By combining (4.1), (4.2), (4.7), recalling that $g_{i}^{\prime}=\left(\alpha_{i} g_{i}-h_{i}\right)$, and taking $\delta=1 / N_{2}$ in (4.7), we obtain, for all $t \geq t_{1}$,

$$
\begin{aligned}
\mathcal{L}^{\prime}(t) & \leq-\left(\frac{l}{2} N_{1}-c\right) \int_{\Omega}\left(|\nabla u|^{2}+|\nabla v|^{2}\right) d x-\left(N_{2} g_{0}-1-N_{1}\right) \int_{\Omega}\left(u_{t}^{2}+v_{t}^{2}\right) d x-\int_{\Omega} F(u, v) d x \\
& +\frac{\alpha_{1}}{2} N\left(g_{1} \circ \nabla u\right)(t)-\left(\frac{1}{2} N-c N_{2}^{2}-C_{\alpha_{1}}\left[c N_{2}^{2}+c N_{1}\right]\right)\left(h_{1} \circ \nabla u\right)(t) \\
& +\frac{\alpha_{2}}{2} N\left(g_{2} \circ \nabla v\right)(t)-\left(\frac{1}{2} N-c N_{2}^{2}-C_{\alpha_{2}}\left[c N_{2}^{2}+c N_{1}\right]\right)\left(h_{2} \circ \nabla v\right)(t)
\end{aligned}
$$


At this point, we choose $N_{1}$ large enough so that

$$
\frac{l}{2} N_{1}-c>4(1-l)
$$

then $N_{2}$ large enough so that

$$
N_{2} g_{0}-1-N_{1}>1 \text {. }
$$

Now, as $g_{i}$ and $-g_{i}^{\prime}$ are positive functions and $\alpha_{i}>0$ we find that

$$
0<\frac{\alpha_{i} g_{i}^{2}(s)}{\alpha_{i} g_{i}(s)-g_{i}^{\prime}(s)}<\frac{\alpha_{i} g_{i}^{2}(s)}{-g_{i}^{\prime}(s)} \Longrightarrow \lim _{\alpha_{i} \rightarrow 0} \frac{\alpha_{i} g_{i}^{2}(s)}{\alpha_{i} g_{i}(s)-g_{i}^{\prime}(s)}=0 \quad \text { for each } s \in[0, \infty)
$$

and also

$$
\frac{\alpha_{i} g_{i}^{2}(s)}{\alpha_{i} g_{i}(s)-g_{i}^{\prime}(s)}<g_{i}(s)
$$

where $g_{i}$ is integrable on $[0, \infty)$, then, by the Lebesgue dominated convergence theorem, we deduce, for $i=1,2$, that

$$
\alpha_{i} C_{\alpha_{i}}=\int_{0}^{\infty} \frac{\alpha_{i} g_{i}^{2}(s)}{\alpha_{i} g_{i}(s)-g_{i}^{\prime}(s)} d s \longrightarrow 0 \quad \text { as } \quad \alpha_{i} \longrightarrow 0 .
$$

Hence, there is $0<\gamma<1$ such that if $\alpha_{i}<\gamma$ then

$$
\alpha_{i} C_{\alpha_{i}}<\frac{1}{8\left[c N_{2}^{2}+c N_{1}\right]} .
$$

Let us choose $N$ large enough and choose $\alpha_{1}, \alpha_{2}$ satisfying

$$
\frac{1}{4} N-c N_{2}^{2}>0 \quad \text { and } \quad \alpha_{1}=\alpha_{2}=\frac{1}{2 N}<\gamma
$$

which means

$$
\frac{1}{2} N-c N_{2}^{2}-C_{\alpha_{i}}\left[c N_{2}^{2}+c N_{1}\right]>0
$$

So, we arrive at

$$
\begin{aligned}
\mathcal{L}^{\prime}(t) \leq & -4(1-l) \int_{\Omega}\left(|\nabla u|^{2}+|\nabla v|^{2}\right) d x-\int_{\Omega}\left(u_{t}^{2}+v_{t}^{2}\right) d x-\int_{\Omega} F(u, v) d x \\
& +\frac{1}{4}\left(g_{1} \circ \nabla u\right)(t)+\frac{1}{4}\left(g_{2} \circ \nabla v\right)(t) .
\end{aligned}
$$

On the other hand, we can easily find, using Young's and Poincaré's inequalities, that

$$
|\mathcal{L}(t)-N E(t)| \leq N_{1}|I(t)|+N_{2}|K(t)| \leq c E(t)
$$

Hence, we can choose $N$ even larger (if needed) so that, for some constants $a_{1}, a_{2}>0$,

$$
a_{1} E(t) \leq \mathcal{L}(t) \leq a_{2} E(t),
$$

that is, (4.15) is satisfied. 


\section{Proof of Theorem 2.2}

We start using (2.10) and (4.1) to conclude that, for any $t \geq t_{1}$,

$$
\begin{aligned}
& \int_{0}^{t_{1}} g_{1}(s) \int_{\Omega}|\nabla u(t)-\nabla u(t-s)|^{2} d x d s+\int_{0}^{t_{1}} g_{2}(s) \int_{\Omega}|\nabla v(t)-\nabla v(t-s)|^{2} d x d s \\
& \leq-\frac{M_{0}}{a} \int_{0}^{t_{1}}\left(g_{1}^{\prime}(s) \int_{\Omega}|\nabla u(t)-\nabla u(t-s)|^{2} d x-g_{2}^{\prime}(s) \int_{\Omega}|\nabla v(t)-\nabla v(t-s)|^{2} d x\right) d s \\
& \leq-c E^{\prime}(t)
\end{aligned}
$$

which can be used in (4.14) and then take $F(t)=\mathcal{L}(t)+c E(t)$, which is clearly equivalent to $E(t)$, to get, for some constant $m>0$ and for all $t \geq t_{1}$,

$$
\begin{aligned}
& \mathcal{L}^{\prime}(t) \leq-4(1-l) \int_{\Omega}\left(|\nabla u|^{2}+|\nabla v|^{2}\right) d x-\int_{\Omega}\left(u_{t}^{2}+v_{t}^{2}\right) d x-\int_{\Omega} F(u, v) d x \\
&+\frac{1}{4}\left(g_{1} \circ \nabla u\right)(t)+\frac{1}{4}\left(g_{2} \circ \nabla v\right)(t) \\
& \leq-m E(t)+c\left(g_{1} \circ \nabla u\right)(t)+c\left(g_{2} \circ \nabla v\right)(t) \\
& \leq-m E(t)-c E^{\prime}(t)+c \int_{t_{1}}^{t} g_{1}(s) \int_{\Omega}|\nabla u(t)-\nabla u(t-s)|^{2} d x d s \\
&+c \int_{t_{1}}^{t} g_{2}(s) \int_{\Omega}|\nabla v(t)-\nabla v(t-s)|^{2} d x d s \\
& \Longrightarrow \quad F^{\prime}(t) \leq-m E(t)+c \int_{t_{1}}^{t} g_{1}(s) \int_{\Omega}|\nabla u(t)-\nabla u(t-s)|^{2} d x d s \\
&+c \int_{t_{1}}^{t} g_{2}(s) \int_{\Omega}|\nabla v(t)-\nabla v(t-s)|^{2} d x d s
\end{aligned}
$$

We consider the following two cases.

(I) $H(t)$ is linear: By Multiplying (5.1) by $\xi(t)=\min \left\{\xi_{1}(t), \xi_{2}(t)\right\}$ and using (H1) and (4.1), we obtain

$$
\begin{aligned}
\xi(t) F^{\prime}(t) \leq & -m \xi(t) E(t)+c \xi(t) \int_{t_{1}}^{t} g_{1}(s) \int_{\Omega}|\nabla u(t)-\nabla u(t-s)|^{2} d x d s \\
& +c \xi(t) \int_{t_{1}}^{t} g_{2}(s) \int_{\Omega}|\nabla v(t)-\nabla v(t-s)|^{2} d x d s
\end{aligned}
$$




$$
\begin{aligned}
& \leq-m \xi(t) E(t)+c \int_{t_{1}}^{t} \xi_{1}(s) g_{1}(s) \int_{\Omega}|\nabla u(t)-\nabla u(t-s)|^{2} d x d s \\
& \quad+c \int_{t_{1}}^{t} \xi_{2}(s) g_{2}(s) \int_{\Omega}|\nabla v(t)-\nabla v(t-s)|^{2} d x d s \\
& \leq-m \xi(t) E(t)-c \int_{t_{1}}^{t} g_{1}^{\prime}(s) \int_{\Omega}|\nabla u(t)-\nabla u(t-s)|^{2} d x d s \\
& \quad-c \int_{t_{1}}^{t} g_{2}^{\prime}(s) \int_{\Omega}|\nabla v(t)-\nabla v(t-s)|^{2} d x d s \\
& \leq-m \xi(t) E(t)-c E^{\prime}(t) .
\end{aligned}
$$

Using the fact that $\xi$ is a nonincreasing continuous function as $\xi_{1}$ and $\xi_{2}$ are nonincreasing, and so $\xi$ is differentiable, with $\xi^{\prime}(t) \leq 0$, for a.e. $t$, then we infer that

$$
(\xi F+c E)^{\prime}(t) \leq \xi(t) F^{\prime}(t)+c E^{\prime}(t) \leq-m \xi(t) E(t), \quad \text { a.e. } t \geq t_{1} .
$$

Hence, using the fact that $\xi F+c E \sim E$, we easily obtain

$$
E(t) \leq c^{\prime} e^{-\bar{c} \int_{t_{1}}^{t} \xi(s) d s} .
$$

(II) $H(t)$ is nonlinear: First, we use Lemma 4.4 and Lemma 4.5 to deduce that

$$
L(t)=\mathcal{L}(t)+M(t)
$$

is nonnegative and it satisfies, for all $t \geq t_{1}$,

$$
\begin{aligned}
L^{\prime}(t) \leq & -(1-l) \int_{\Omega}\left(|\nabla u|^{2}+|\nabla v|^{2}\right) d x-\int_{\Omega}\left(u_{t}^{2}+v_{t}^{2}\right) d x-\int_{\Omega} F(u, v) d x \\
& -\frac{1}{4}\left(g_{1} \circ \nabla u\right)(t)-\frac{1}{4}\left(g_{2} \circ \nabla v\right)(t) \\
\leq & -B E(t)
\end{aligned}
$$

where $B$ is some positive constant. Therefore

$$
B \int_{t_{1}}^{t} E(s) d s \leq L\left(t_{1}\right)-L(t) \leq L\left(t_{1}\right)
$$

which implies that

$$
\int_{0}^{\infty} E(s) d s<\infty \text {. }
$$


Hence, by noting that

$$
\begin{aligned}
I_{1}(t)+I_{2}(t) & =q \int_{t_{1}}^{t} \int_{\Omega}|\nabla u(t)-\nabla u(t-s)|^{2} d x d s+q \int_{t_{1}}^{t} \int_{\Omega}|\nabla v(t)-\nabla v(t-s)|^{2} d x d s \\
& \leq c q \int_{0}^{t} E(s) d s,
\end{aligned}
$$

(5.2) allows for a constant $0<q<1$ chosen so that, for all $t \geq t_{1}$,

$$
I_{i}(t)<1 \text {. }
$$

We also assume, without loss of generality, $t_{1}$ large enough so that $I_{i}\left(t_{1}\right)>0$ which mean $I_{i}(t)>0$ for all $t \geq t_{1}$. Also, we define $\lambda(t)$ by

$$
\lambda(t):=-\int_{t_{1}}^{t} g_{1}^{\prime}(s) \int_{\Omega}|\nabla u(t)-\nabla u(t-s)|^{2} d x d s,
$$

and observe that $\lambda(t) \leq-c E^{\prime}(t)$. Since $H$ is strictly convex on $(0, r]$ and $H(0)=0$, then

$$
H(\theta x) \leq \theta H(x)
$$

provided $0 \leq \theta \leq 1$ and $x \in(0, r]$. The use of this fact, hypothesis (H1), (5.3), and Jensen's inequality leads to

$$
\begin{aligned}
\lambda(t) & =\frac{1}{q I_{1}(t)} \int_{t_{1}}^{t} I_{1}(t)\left(-g_{1}^{\prime}(s)\right) \int_{\Omega} q|\nabla u(t)-\nabla u(t-s)|^{2} d x d s \\
& \geq \frac{1}{q I_{1}(t)} \int_{t_{1}}^{t} I_{1}(t) \xi_{1}(s) H\left(g_{1}(s)\right) \int_{\Omega} q|\nabla u(t)-\nabla u(t-s)|^{2} d x d s \\
& \geq \frac{\xi(t)}{q I_{1}(t)} \int_{t_{1}}^{t} H\left(I_{1}(t) g_{1}(s)\right) \int_{\Omega} q|\nabla u(t)-\nabla u(t-s)|^{2} d x d s \\
& \geq \frac{\xi(t)}{q} H\left(\frac{1}{I_{1}(t)} \int_{t_{1}}^{t} I_{1}(t) g_{1}(s) \int_{\Omega} q|\nabla u(t)-\nabla u(t-s)|^{2} d x d s\right) \\
& =\frac{\xi(t)}{q} H\left(q \int_{t_{1}}^{t} g_{1}(s) \int_{\Omega}|\nabla u(t)-\nabla u(t-s)|^{2} d x d s\right) \\
& =\frac{\xi(t)}{q} \bar{H}\left(q \int_{t_{1}}^{t} g_{1}(s) \int_{\Omega}|\nabla u(t)-\nabla u(t-s)|^{2} d x d s\right)
\end{aligned}
$$


where $\bar{H}$ is an extension of $H$ such that $\bar{H}$ is strictly increasing and strictly convex $C^{2}$ function on $(0, \infty)$ [see (2.11)]. This implies that

$$
\int_{t_{1}}^{t} g_{1}(s) \int_{\Omega}|\nabla u(t)-\nabla u(t-s)|^{2} d x d s \leq \frac{1}{q} \bar{H}^{-1}\left(\frac{q \lambda(t)}{\xi(t)}\right) .
$$

We also define

$$
\chi(t):=-\int_{t_{1}}^{t} g_{2}^{\prime}(s) \int_{\Omega}|\nabla v(t)-\nabla v(t-s)|^{2} d x d s .
$$

and repeat the above steps to get

$$
\int_{t_{1}}^{t} g_{2}(s) \int_{\Omega}|\nabla v(t)-\nabla v(t-s)|^{2} d x d s \leq \frac{1}{q} \bar{H}^{-1}\left(\frac{q \chi(t)}{\xi(t)}\right) .
$$

Therefore, using the properties of $\bar{H},(5.1)$ becomes

$$
\begin{aligned}
F^{\prime}(t) & \leq-m E(t)+c \bar{H}^{-1}\left(\frac{q \lambda(t)}{\xi(t)}\right)+c \bar{H}^{-1}\left(\frac{q \chi(t)}{\xi(t)}\right) \\
& \leq-m E(t)+c \bar{H}^{-1}\left(\frac{q \lambda(t)}{\xi(t)}+\frac{q \chi(t)}{\xi(t)}\right), \quad \forall t \geq t_{1} .
\end{aligned}
$$

Now, for $\varepsilon_{0}<r$, using (5.4), and the fact that $E^{\prime} \leq 0, \bar{H}^{\prime}>0, \bar{H}^{\prime \prime}>0$, we find that the functional $F_{1}$, defined by

$$
F_{1}(t):=\bar{H}^{\prime}\left(\varepsilon_{0} \frac{E(t)}{E(0)}\right) F(t)+E(t)
$$

is equivalent to $E$ and

$$
\begin{aligned}
& F_{1}^{\prime}(t)=\varepsilon_{0} \frac{E^{\prime}(t)}{E(0)} \bar{H}^{\prime \prime}\left(\varepsilon_{0} \frac{E(t)}{E(0)}\right) F(t)+\bar{H}^{\prime}\left(\varepsilon_{0} \frac{E(t)}{E(0)}\right) F^{\prime}(t)+E^{\prime}(t) \\
& \leq-m E(t) \bar{H}^{\prime}\left(\varepsilon_{0} \frac{E(t)}{E(0)}\right)+c \bar{H}^{\prime}\left(\varepsilon_{0} \frac{E(t)}{E(0)}\right) \bar{H}^{-1}\left(\frac{q \lambda(t)}{\xi(t)}+\frac{q \chi(t)}{\xi(t)}\right)+E^{\prime}(t) .
\end{aligned}
$$

Let $\bar{H}^{*}$ be the convex conjugate of $\bar{H}$ in the sense of Young (see [4] p. 61-64), then

$$
\bar{H}^{*}(s)=s\left(\bar{H}^{\prime}\right)^{-1}(s)-\bar{H}\left[\left(\bar{H}^{\prime}\right)^{-1}(s)\right]
$$

and $\bar{H}^{*}$ satisfies the following Young's inequality

$$
A B \leq \bar{H}^{*}(A)+\bar{H}(B) .
$$


With $A=\bar{H}^{\prime}\left(\varepsilon_{0} \frac{E(t)}{E(0)}\right)$ and $B=\bar{H}^{-1}\left(\frac{q \lambda(t)}{\xi(t)}+\frac{q \chi(t)}{\xi(t)}\right)$, using (4.1) and (5.5)-(5.7), we arrive at

$$
\begin{aligned}
F_{1}^{\prime}(t) & \leq-m E(t) \bar{H}^{\prime}\left(\varepsilon_{0} \frac{E(t)}{E(0)}\right)+c \bar{H}^{*}\left(\bar{H}^{\prime}\left(\varepsilon_{0} \frac{E(t)}{E(0)}\right)\right)+c \frac{q \lambda(t)}{\xi(t)}+c \frac{q \chi(t)}{\xi(t)}+E^{\prime}(t) \\
& \leq-m E(t) \bar{H}^{\prime}\left(\varepsilon_{0} \frac{E(t)}{E(0)}\right)+c \varepsilon_{0} \frac{E(t)}{E(0)} \bar{H}^{\prime}\left(\varepsilon_{0} \frac{E(t)}{E(0)}\right)+c \frac{q \lambda(t)}{\xi(t)}+c \frac{q \chi(t)}{\xi(t)}+E^{\prime}(t) .
\end{aligned}
$$

Then, we multiply by $\xi(t)$ and use the fact that, as $\varepsilon_{0} \frac{E(t)}{E(0)}<r, \bar{H}^{\prime}\left(\varepsilon_{0} \frac{E(t)}{E(0)}\right)=H^{\prime}\left(\varepsilon_{0} \frac{E(t)}{E(0)}\right)$ to get

$$
\begin{aligned}
\xi(t) F_{1}^{\prime}(t) \leq & -m \xi(t) E(t) H^{\prime}\left(\varepsilon_{0} \frac{E(t)}{E(0)}\right)+c \varepsilon_{0} \frac{E(t)}{E(0)} \xi(t) H^{\prime}\left(\varepsilon_{0} \frac{E(t)}{E(0)}\right) \\
& +c q \lambda(t)+c q \chi(t)+\xi(t) E^{\prime}(t) \\
\leq & -m \xi(t) E(t) H^{\prime}\left(\varepsilon_{0} \frac{E(t)}{E(0)}\right)+c \varepsilon_{0} \frac{E(t)}{E(0)} \xi(t) H^{\prime}\left(\varepsilon_{0} \frac{E(t)}{E(0)}\right)-c E^{\prime}(t)
\end{aligned}
$$

Consequently, with $F_{2}=\xi F_{1}+c E$, which satisfies, for some $\alpha_{1}, \alpha_{2}>0$,

$$
\alpha_{1} F_{2}(t) \leq E(t) \leq \alpha_{2} F_{2}(t)
$$

and with a suitable choice of $\varepsilon_{0}$, we obtain, for some constant $k>0$ and for all $t \geq t_{1}$,

$$
F_{2}^{\prime}(t) \leq-k \xi(t)\left(\frac{E(t)}{E(0)}\right) H^{\prime}\left(\varepsilon_{0} \frac{E(t)}{E(0)}\right)=-k \xi(t) H_{2}\left(\frac{E(t)}{E(0)}\right)
$$

where $H_{2}(t)=t H^{\prime}\left(\varepsilon_{0} t\right)$.

Since $H_{2}^{\prime}(t)=H^{\prime}\left(\varepsilon_{0} t\right)+\varepsilon_{0} t H^{\prime \prime}\left(\varepsilon_{0} t\right)$, then, using the strict convexity of $H$ on $(0, r]$, we find that $H_{2}^{\prime}(t)$, $H_{2}(t)>0$ on $(0,1]$. Thus, with

$$
R(t)=\frac{\alpha_{1} F_{2}(t)}{E(0)}
$$

taking in account (5.8) and (5.9), we have

$$
R(t) \sim E(t)
$$

and, for some $k_{1}>0$,

$$
R^{\prime}(t) \leq-k_{1} \xi(t) H_{2}(R(t)), \quad \forall t \geq t_{1}
$$

Then, the integration over $\left(t_{1}, t\right)$ yields

$$
\begin{aligned}
& \int_{t_{1}}^{t} \frac{-R^{\prime}(s)}{H_{2}(R(s))} d s \geq k_{1} \int_{t_{1}}^{t} \xi(s) d s \Longrightarrow \int_{\varepsilon_{0} R(t)}^{\varepsilon_{0} R\left(t_{1}\right)} \frac{1}{s H^{\prime}(s)} d s \geq k_{1} \int_{t_{1}}^{t} \xi(s) d s \\
& \Longrightarrow R(t) \leq \frac{1}{\varepsilon_{0}} H_{1}^{-1}\left(k_{1} \int_{t_{1}}^{t} \xi(s) d s\right),
\end{aligned}
$$


where $H_{1}(t)=\int_{t}^{r} \frac{1}{s H^{\prime}(s)} d s$. Here, we have used, based on the properties of $H$, the fact that $H_{1}$ is strictly decreasing function on $(0, r]$ and $\lim _{t \rightarrow 0} H_{1}(t)=+\infty$. A combination of (5.10) and (5.11), estimate (2.7) is established.

\section{Acknowledgment}

This work was supported by MASEP Research Group in the Research Institute of Sciences and Engineering at University of Sharjah.

\section{Bibliography}

[1] Alabau-Boussouira F. and Cannarsa P., A general method for proving sharp energy decay rates for memory dissipative evolution equations, C. R. Acad. Sci. Paris Ser. I 347 (2009), 867-872.

[2] Alabau-Boussouira F., Cannarsa P. and Sforza D., Decay estimates for the second order evolution equation with memory, J. Funct. Anal. 245 (2008), 1342-1372.

[3] Andrade D. and Mognon A., Global solutions for a system of Klein-Gordon equations with memory, Bol. Soc. Paran. Mat. 21 (1/2) (2003), 127-138.

[4] Arnold V.I., Mathematical Methods of Classical Mechanics, Springer-Verlag, New York,1989.

[5] Barreto R. and Munoz Rivera J.E., Uniform rates of decay in nonlinear viscoelasticity for polynomial decaying kernels, Appl. Anal. 60 (1996), 263-283.

[6] Cannarsa P. and Sforza D., Integro-differential equations of hyperbolic type with positive definite kernels, J. Differential Equations 250 (2011), 4289-4335.

[7] Cavalcanti M.M., Domingos Cavalcanti V.N., Lasiecka I. and Nascimento F.A., Intrinsic decay rate estimates for the wave equation with competing viscoelastic and frictional dissipative effects, Discrete Contin. Dyn. Syst. Ser. B 19 (7) (2014), 1987-2012.

[8] Cavalcanti M.M., Domingos Cavalcanti V.N., Lasiecka I. and Webler C.M., Intrinsic decay rates for the energy of a nonlinear viscoelastic equation modeling the vibrations of thin rods with variable density, Adv. Nonlinear Anal. 6(2) (2017), 121-145.

[9] Cavalcanti M. M. and Oquendo H. P., Frictional versus viscoelastic damping in a semilinear wave equation, SIAM J. Control Optim. 42 (4) (2003), 1310-1324.

[10] Guesmia A., Asymptotic stability of abstract dissipative systems with infinite memory, J. Math. Anal. Appl. 382 (2011), 748-760.

[11] Han X. and Wang M., General decay of energy for a viscoelastic equation with nonlinear damping, Math. Methods Appl. Sci. 32 (3) (2009), 346-358.

[12] Hao J. and Cai L., Uniform decay of solutions for coupled viscoelastic wave equations, Electron. J. Differential Equations 2016 (72) (2016), 1-11. 
[13] Lasiecka I., Messaoudi S.A., and Mustafa M.I., Note on intrinsic decay rates for abstract wave equations with memory, J. Math. Phys. 54, 031504 (2013); doi: 10.1063/1.4793988.

[14] Lasiecka I. and Wang X., Intrinsic decay rate estimates for semilinear abstract second order equations with memory, New prospects in direct, inverse and control problems for evolution equations, 271303, Springer INdAM Ser., 10, Springer, Cham, 2014.

[15] Liu W.J., General decay of solutions of a nonlinear system of viscoelastic equations, Acta Appl. Math. 110 (2010), 153-165.

[16] Liu W.J., General decay rate estimate for a viscoelastic equation with weakly nonlinear timedependent dissipation and source terms, J. Math. Phys 50 (11) (2009), art. No 113506.

[17] Liu W.J., Uniform decay of solutions for a quasilinear system of viscoelastic equations, Nonlinear Anal. 71 (2009), 2257-2267.

[18] Liu W.J., Chen Z.J. and Chen D.Q., New general decay results for a Moore-Gibson-Thompson equation with memory, Appl. Anal. (2020), 99:15, 2622-2640, DOI: 10.1080/00036811.2019.1577390.

[19] Liu W.J., Chen Z.J. and Tu Z., New general decay result for a fourth-order Moore-Gibson-Thompson equation with memory, Electronic Research Archive 28 (1) (2020), 433-457.

[20] Messaoudi S.A. General decay of solutions of a viscoelastic equation, J. Math. Anal. Appl. 341 (2008), 1457-1467.

[21] Messaoudi S.A., General decay of the solution energy in a viscoelastic equation with a nonlinear source, Nonlinear Anal. 69 (2008), 2589-2598.

[22] Messaoudi S.A. and Al-Gharabli M.M., A general decay result of a nonlinear system of wave equations with infinite memories, Appl. Math. Computations 259 (2015), 540-551.

[23] Messaoudi S.A. and Mustafa M.I., A stability result in a memory-type Timoshenko system, Dynam. Systems Appl. 18 (2009), 457-468.

[24] Messaoudi S.A. and Tatar N.-e,, Exponential and polynomial decay for a quasilinear viscoelastic equation, Nonlinear Anal. TMA 68 (2007), 785-793.

[25] Messaoudi S.A. and Tatar N.-e, Uniform stabilization of solutions of a nonlinear system of viscoelastic equations, Appl. Anal. 87 (3) (2008), 247-263.

[26] Munoz Rivera J.E., Asymptotic behavior in linear viscoelasticity, Quart. Appl. Math. 52 (4) (1994), 628-648.

[27] Munoz Rivera J.E. and Oquendo H. P., Exponential stability to a contact problem of partially viscoelastic materials, J. Elasticity 63 (2) (2001), 87-111.

[28] Munoz Rivera J.E. and Salvatierra A. P., Asymptotic behavior of the energy in partially viscoelastic materials, Quart. Appl. Math. 59 (3) (2001), 557-578.

[29] Munoz Rivera J.E., Naso M.G. and Vegni F.M., Asymptotic behavior of the energy for a class of weakly dissipative second-order systems with memory, J. Math. Anal. Appl. 286 (2) (2003), 692-704. 
[30] Munoz Rivera J.E. and Naso M.G., On the decay of the energy for systems with memory and indefinite dissipation, Asympt. Anal. 49 (3-4) (2006), 189-204.

[31] Mustafa M.I., Optimal decay rates for the viscoelastic wave equation, Math. Methods Appl. Sci. 41 (1) (2018), 192-204.

[32] Mustafa M.I., Uniform decay rates for viscoelastic dissipative systems, J. Dyn. Control Syst. 22 (1) (2016), 101-116.

[33] Mustafa M.I., Well posedness and asymptotic behavior of a coupled system of nonlinear viscoelastic equations, Nonlinear Anal. RWA 13 (2012), 452-463.

[34] Mustafa M.I. and Messaoudi S.A., General stability result for viscoelastic wave equations, J. Math. Phys 53, 053702 (2012); doi: 10.1063/1.4711830.

[35] Park J. and Park S., General decay for quasilinear viscoelastic equations with nonlinear weak damping, J. Math. Physics 50 (8), art. No 083505 (2009).

[36] Said-Houari B., Messaoudi S.A. and Guesmia A., General decay of solutions of a nonlinear system of viscoelastic wave equations, Nonlinear Differ. Equ. Appl. 18 (6) (2011), 659-684.

[37] Santos M.L., Decay rates for solutions of a system of wave equations with memory, Electron. J. Differential Equations 2002 (38) (2002), 1-17.

[38] Wu S.T., General decay for a wave equation of Kirchhoff type with a boundary control of memory type, Boundary Value Problems 2011 (2011), 15pp. 